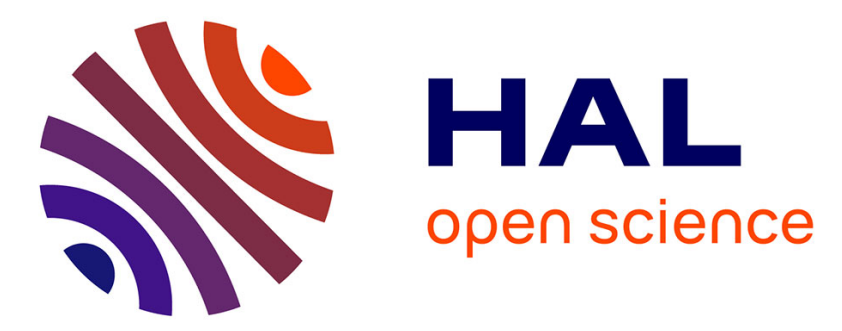

\title{
Compressional stability of optical fibres: a combined experimental and computer study
}

Lukas Capek, Petr Henys, Vincent Placet, Brigita Kolcavova

\section{To cite this version:}

Lukas Capek, Petr Henys, Vincent Placet, Brigita Kolcavova. Compressional stability of optical fibres: a combined experimental and computer study. Journal of Materials Science, 2018, 53 (21), pp.1 - 8. hal-02130047

\section{HAL Id: hal-02130047 https://hal.science/hal-02130047}

Submitted on 15 May 2019

HAL is a multi-disciplinary open access archive for the deposit and dissemination of scientific research documents, whether they are published or not. The documents may come from teaching and research institutions in France or abroad, or from public or private research centers.
L'archive ouverte pluridisciplinaire HAL, est destinée au dépôt et à la diffusion de documents scientifiques de niveau recherche, publiés ou non, émanant des établissements d'enseignement et de recherche français ou étrangers, des laboratoires publics ou privés. 
See discussions, stats, and author profiles for this publication at: https://www.researchgate.net/publication/326304562

Compressional stability of optical fibres: a combined experimental and computer study

Article in Journal of Materials Science · July 2018

DOI: 10.1007/s 10853-018-2678-5

CITATIONS

4 authors, including:

20

Lukas Capek

Technical University of Liberec

52 PUBLICATIONS 151 CITATIONS

SEE PROFILE

Vincent Placet

University of Franche-Comté

112 PUBLICATIONS $\mathbf{7 3 0}$ CITATIONS

SEE PROFILE
Petr Henyš

Technical University of Liberec

29 PUBLICATIONS 26 CITATIONS

SEE PROFILE

Some of the authors of this publication are also working on these related projects:

Modelling musical instruments View project

STRUTEX - One Day Course on Finite Element Method View project 


\title{
Compressional stability of optical fibres: a combined experimental and computer study
}

\author{
Lukas Capek • Petr Henys • Vincent \\ Placet • Brigita Kolcavova
}

Received: date / Accepted: date

\begin{abstract}
Compressional stability is an important mechanical factor that influences the overall performance of a fibre. Assessment of the compressive stability and internal-stress distribution of a fibre must be performed to determine the effect of stress-induced birefringence due to the high deformation gradients in an unstable fibre. The purpose of this study is to combine experimental and finite element investigations of stress evolution and large post-buckling fibre deformations. The results of this study show that stability is greatly influenced by the initial curvature of the fibre. Increasing the deformation of the fibre leads to post-buckling behaviour that results in a fibre taking a complex shape with altered stress-field components. The results are compared with the analytical relation given by Euler's theory of a thin beam and show that the simple analytical formula cannot predict the complicated post-buckling states and stresses.
\end{abstract}

Keywords finite element $\cdot$ birefringence $\cdot$ buckling $\cdot$ fibre $\cdot$ large deformation

\section{Introduction}

The development of optical fibres was one of the most significant advances in communication systems during the twentieth century. Optical fibres offer many

Lukas Capek, Petr Henys(corresponding author)

Technical University of Liberec, Department of Technologies and Structures, Studentska 2, 46117 Liberec, Czech Republic

Tel.: +420-48-5353257

E-mail: petr.henys@tul.cz

Vincent Placet

University Bourgogne Franche-Comte, FEMTO-ST Institute, 24 rue de l'Epitaphe 25030, Besancon, France

Tel.: +03-81-6660557

Brigita Kolcavova

Technical University of Liberec, Department of Technologies and Structures, Studentska 2, 46117 Liberec, Czech Republic

Tel.: +420-48-5353274 
benefits in comparison to classical conductors, such as high speed transmission and less attenuation. In the late 1980s, as optical fibres were being improved, their use spread into a wide range of applications. One promising application involves smart textile development, where the optical fibres are incorporated into the woven structure of a fabric [1]. However, regardless of their intended application, the fundamental questions of mechanical reliability and the nature of failure modes must be addressed. Neither the fabrication process nor the ultimate use should change the signal propagation or damage the fibre structure. In the literature, it is generally presumed that fibres are dominantly loaded in tension; thus, the strength of an optical fibre has usually been measured under uniaxial tension [4-7. However, if an optical fibre is incorporated into a stratified structure, the mechanism of loading will be different. Both the weaving process and subsequent packing causes bending and buckling of the textile structure [8, 9]. Cheng et al. demonstrated that the strength of an optical fibre under tensile testing is smaller than that determined in a two-point bending test [10. Another type of failure is buckling, in which investigators seek to determine the maximum load that a structure can support before it collapses. The collapse is not related to material yield but is instead governed by the material and the geometric stiffness of the column. The basic linear elastic theory solution is provided by Eulers beam-bending equation. In the EulerBernoulli beam theory, shear deformations are neglected, and plane sections remain plane and normal to the longitudinal axis. In the 1960s, Timoshenko proposed a new formulation of beam theory, in which plane sections still remain plane but are no longer normal to the longitudinal axis [11. The difference between the normal to the axis and the rotated plane section constitutes shear deformation. However, limited research has been conducted on fibre- or yarn-buckling, although there have been some attempts to improve Timoshenko's equation to consider the suppleness of the fibres [12. The stress strain relation during the collapse state is different from classical compression state and we hypothese that one of the stress component would be dominant from the other one. The aim of this work is to show, how the Euler's beam formula is applicable for buckling of optical fibres and what is the stress state of buckled optical fibre.

\section{Material and Methods}

We chose two different approaches to determine the stability of the optical fibres. We first performed a set of experiments to determine the buckling states of an optical fibre. To obtain further insight into the post-buckling states, we conducted a finite element computer study. We subsequently compared the experimental and numerical results with the analytical Euler formula as follows:

$$
F_{\text {crit }}=4 \pi^{2} E I \frac{1}{L^{2}}
$$

where $F_{c r i t}$ is the critical force at which a beam buckles, $E$ is Young's modulus, $I$ is the second moment of area and $L$ is the length of the sample. 
Table 1 The opto-mechanical properties of tested fibre.

$\begin{array}{ll}\text { Core: index of refraction [-] } & 1.49 \\ \text { Skin: index of refraction [-] } & 1.42 \\ \text { Numerical aperture [-] } & 0.44 \\ \text { Acceptance [ }{ }^{\circ} \text { ] } & 52.2 \\ \text { Tension strength [MPa] } & 78\end{array}$

\subsection{Experiment Setup}

We tested optical fibres with diameters of $0.75 \mathrm{~mm}$, each of which comprise a polymethylmethacrylate core (PMMA) and a polycarbonate skin. The opto-mechanical properties of the fibres are listed in Table 1, and the cross-sectional views of the tested fibres are shown in Figure 1. We inspected the chemical compositions and cross-sectional surfaces of the samples using energy-dispersive X-ray spectroscopy (EDX) and scanning electron microscopy (SEM). The samples were sorted into groups of 20. Fibres of different lengths, ranging from $5 \mathrm{~mm}$ to $100 \mathrm{~mm}$, with 5 -mm increments, were loaded at a stretch rate of $20 \mathrm{~mm} / \mathrm{min}$ until loss of the stability. A schematic of the experimental setup is shown in Figure 2 . The compression tests were performed using a universal machine (Testometric M350-5CT) with a $100 \mathrm{~N}$ force gauge, and we determined the critical force, post-buckling geometry and functionality of each fibre.

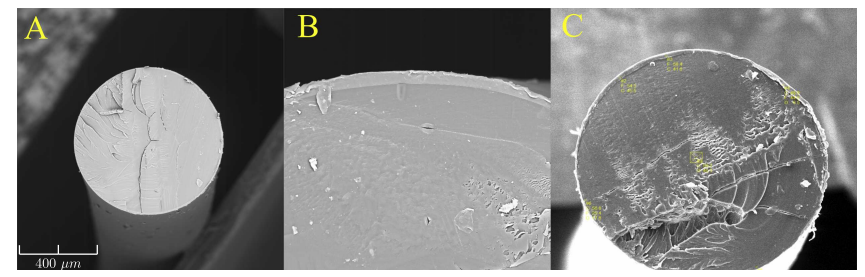

Fig. 1 A cross-sectional view SEM (A-B) and EDX (C) of optical fibre taken by SEM.

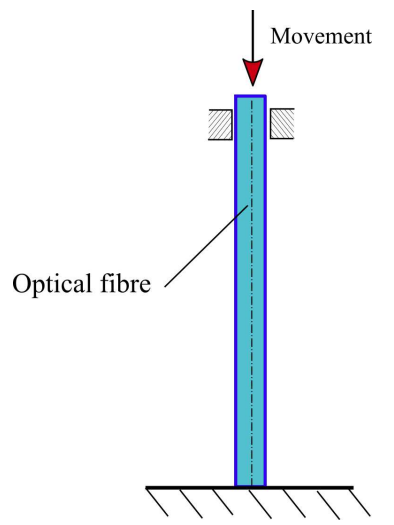

Fig. 2 Experimental set-up: optical fibre constrained at bottom end. 


\subsection{Finite Element Model}

The geometry of a fibre was discretised using linear tetrahedral elements (CGAL library [13]). The displacement $\mathbf{u}$ and its gradient $\nabla \mathbf{u}$ were discretised on tetrahedra using a continuous, isoparametric, quadratic approximation (Lagrange-P2). However, the gradient $\nabla \mathbf{u}$ was further projected onto the discontinuous space DG-0 to obtain sufficient resolution of the fibre cross-section. We used the GreenLagrange strain tensor $\mathrm{C}$ and the second Piola-Kirchoff stress tensor P for large deformation(total lagrangian framework); both are second-order symmetric tensors. Convergence analysis showed the optimal spacing of the radial elements to be $\frac{r}{15}$, where $r$ is the radius of the fibre, in order for the relative error in $\mathrm{H}^{1}$ to be less than $5 \%$ in terms of the stress tensor norm. The material model we used is based on the Saint Venant-Kirchhoff model written in terms of the strain energy:

$$
W(C)=\frac{\lambda}{2} \operatorname{tr}(C)^{2}+\mu \operatorname{tr}\left(C^{2}\right)
$$

where $\lambda$ and $\mu$ are the Lam constants, which are given in terms of the Young's modulus and Poisson's ratio:

$$
\lambda=\frac{E \nu}{(1+\nu)(1-2 \nu)} ; \mu=\frac{E}{2(1+\nu)}
$$

We determined these material constants from auxiliary uniaxial experiments. We used the arc-length continuation method to determine the stability points [14. The underlying linear solver is based on the Krylov solver (using the conjugate gradient method and the algebraic, multigrid preconditioner CG-AMG (PETSc/Hypre) [15] [16]). We perturbed the initial beam geometry with the small parameter $\varepsilon \in\left\{1^{-5}-1^{-10}\right\}$ to introduce a geometrical imperfection. We implemented the perturbation as a half-sine spatial function to mimic the first perturbation mode known from the auxiliary eigenvalue analysis:

$$
f(x)=\sin \left(\frac{\pi}{L} x\right)
$$

The eigenvalue analysis of the underlying system is intended to determine the three modes with the longest wavelengths for which buckling can occur. We used the Locally optimal block preconditioned conjugate gradient method (SLEPc) 17 to find the smallest eigenvalues and corresponding eigenvectors of the Hermitian system. Convergence analysis again showed that the mesh spacing must be $\frac{r}{15}$ to obtain a relative error less than $5 \%$. The computational framework is based on the problem-solving environment library Fenics 2017.1 [18].

\section{Results}

\subsection{Experimental Results}

We used a compression test to determine the post-buckling state and corresponding critical force on the optical fibre. The critical force is the maximal force that can be applied to a fibre before it suddenly decreases; this force drop occurred up to sample lengths of $35 \mathrm{~mm}$. For long samples, instead of a force decrease, we 


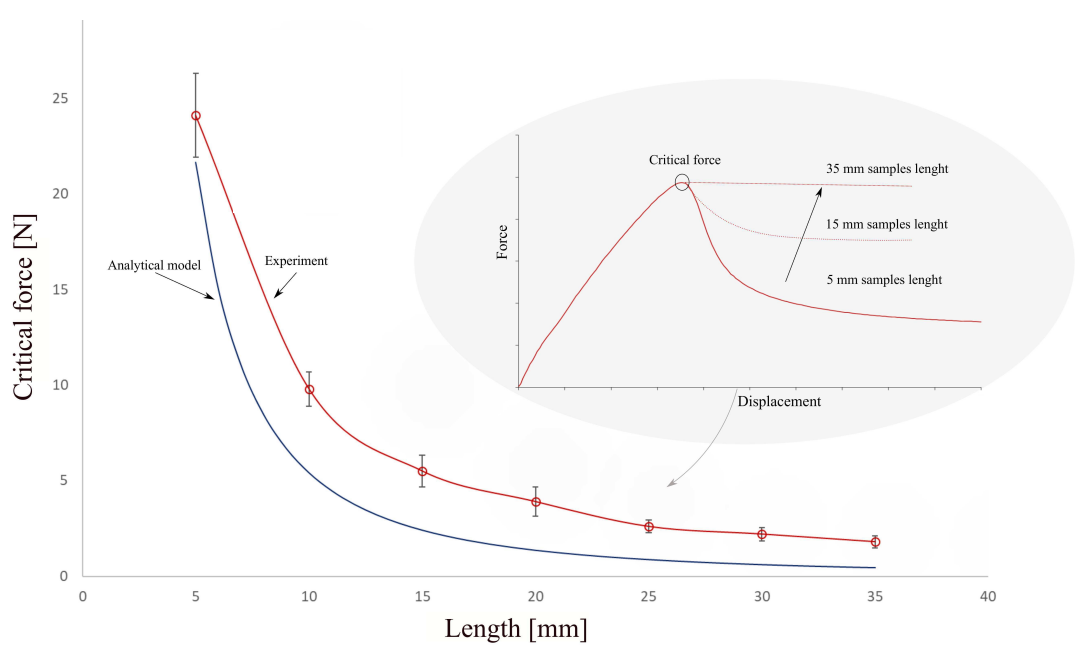

Fig. 3 The critical force as a function of samples length.

observed a horizontal force distribution. The critical force as a function of sample length is shown in Figure 3 . The difference between the experimentally measured critical force and the analytical Euler result increases linearly with the sample length, with a maximal difference of $309 \%$, as shown in Figure 4 There are two

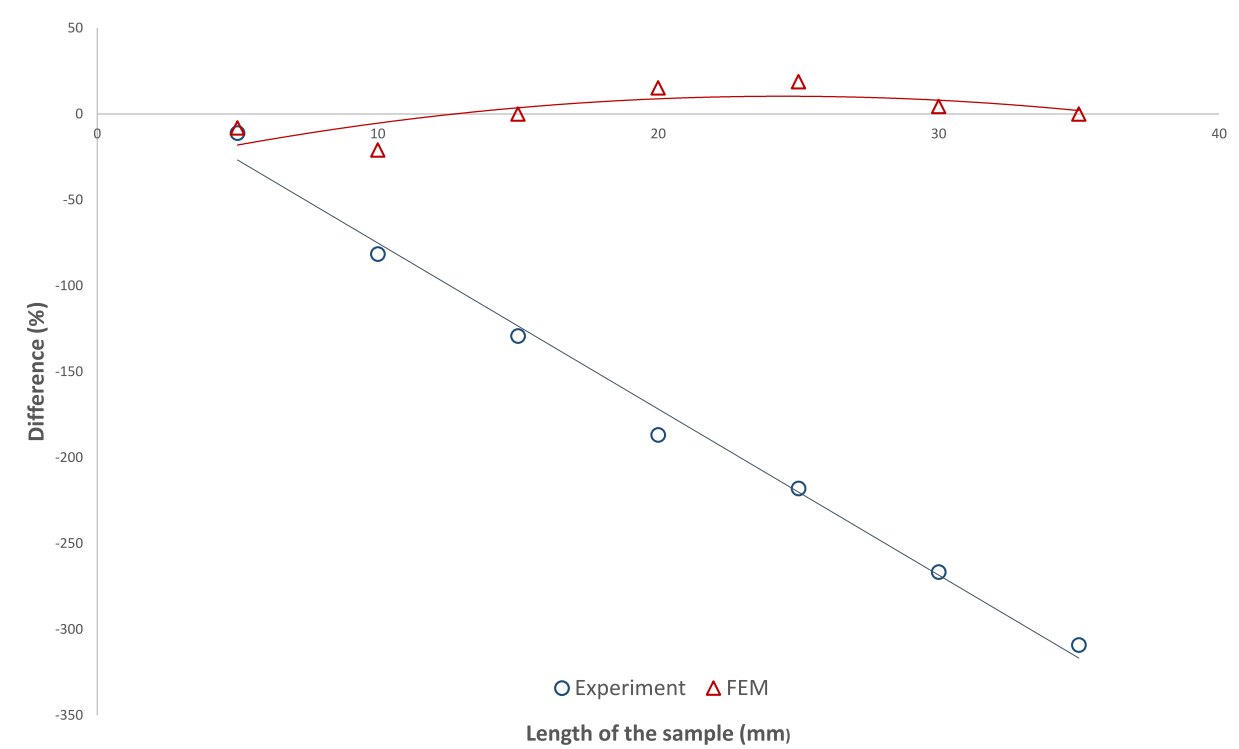

Fig. 4 The difference (\%) between the analytical, numerical and experimental result depending on samples length. 
principal post-buckling shapes that depend on the sample length. Up to length 95 $\mathrm{mm}$, the post-buckling shape is typically a V shape, as shown in Figure 5(A). For long fibres, the shape changes to a spiral, as shown in Figure 5.

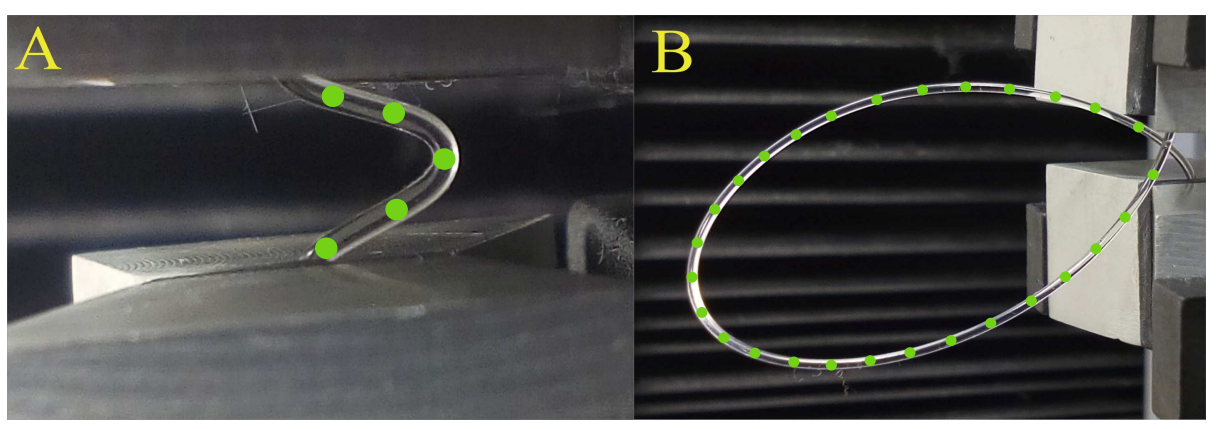

Fig. 5 The post buckling shapes, A) from 5 to 95, B) higher than $95 \mathrm{~mm}$. The green dots follow the fibre deformation shape for better visibility.

\subsection{Computational analyses results}

The three buckling modes with the longest wavelengths are shown in Figure 6 . From our experiments, the critical forces for the first buckling mode alone are -22.3 ,

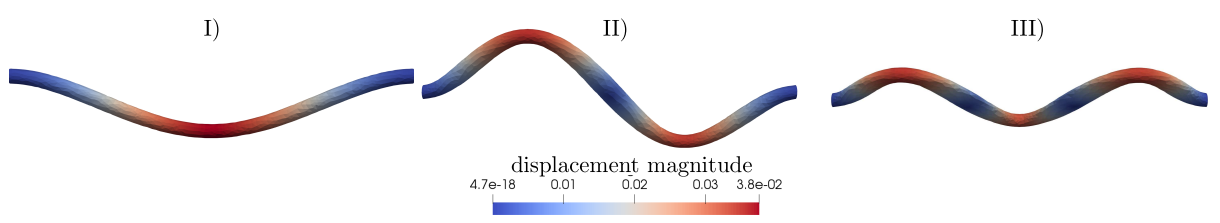

Fig. 6 Three stability modes of the fibre(an example with $\mathrm{L}=20 \mathrm{~mm}$ ).

$-8.1,-5.5,-4.6,-3.2,-2.3$ and $-1.8 \mathrm{~N}$ for the respective fibre lengths ranging from 5 to $35 \mathrm{~mm}$. Up to this threshold, the displacement-force relation is approximately linear. Beyond the threshold, the force decreases up to a limiting value depending mainly on bending stiffness of the fibre. Further increasing the load causes the postbuckling state to evolve, as shown in Figure 7. This occurs for fibres longer than the threshold length $\mathrm{L}>35 \mathrm{~mm}$, as shown experimentally. Figure 7 shows that that the shape of the fibre loop becomes complex after it is deformed to around $75 \%$ of its length. Force switches from being a compressional force to being a tension force. The fibre does not stretch further after passing the zero-force state. The final deformation becomes a ring in the plane XZ. Figure 8 shows the variations in the components of the stress tensor along the length of the fibre. The stress value $-96 \mathrm{MPa}$, which is the largest in magnitude, occurs in the component $\sigma_{x x}$ at the initial state, and the magnitude of the stress decreases to $-58 \mathrm{MPa}$ in the final state. The normal components $\sigma_{x x}, \sigma_{y y}, \sigma_{z z}$ tend to decrease toward the final 


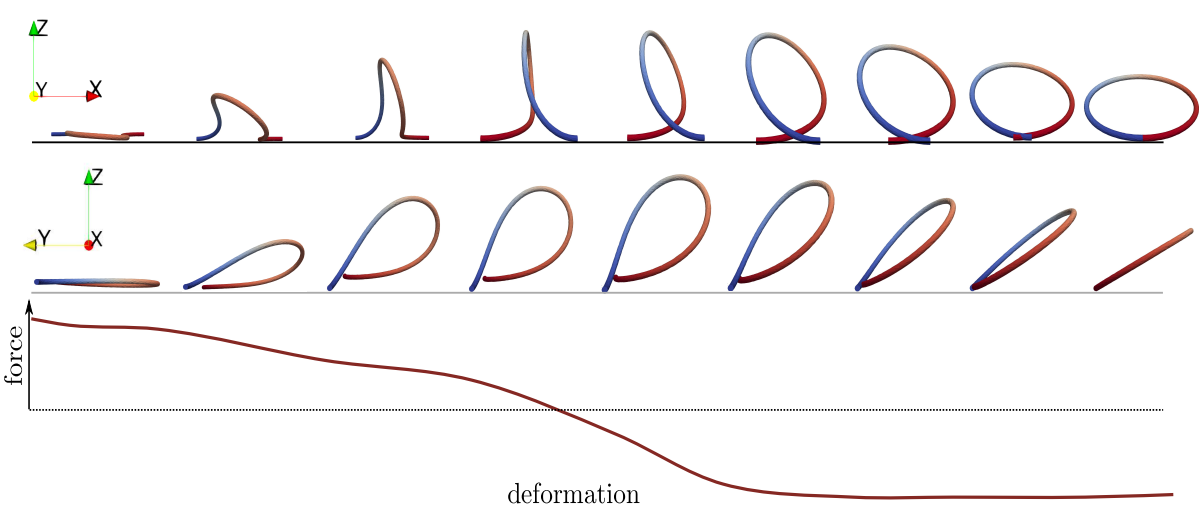

Fig. 7 The evolution of fibre deformation state at post buckling domain. The bottom chart of force[N]-deformation[mm] curve shows a change of sign during the post-buckling state.

state, while the shear components $\sigma_{x y}, \sigma_{x z}$ tend to increase in value up to the final state.
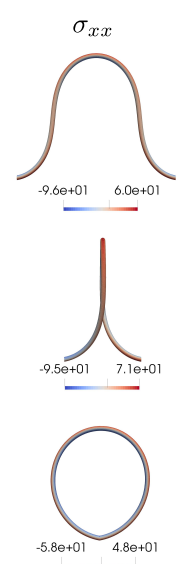
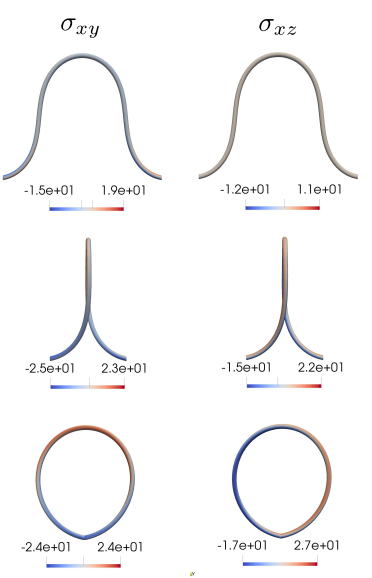

$\therefore$
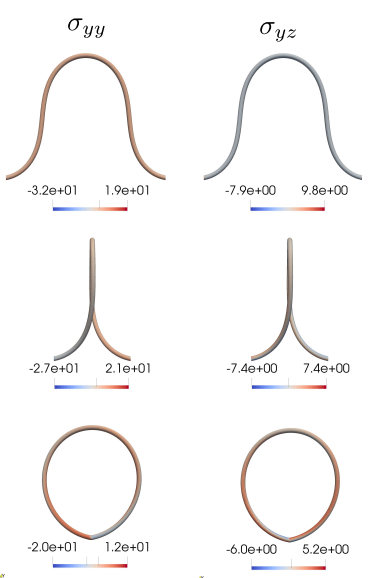
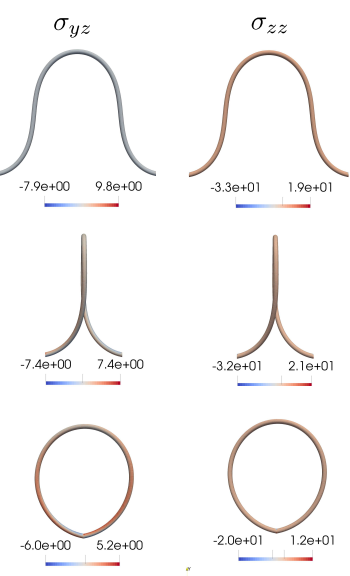

Fig. 8 Stress tensor components evaluation [MPa] at three fibre states $(\mathrm{L}=50 \mathrm{~mm})$ : bottom - final state; middle-middle state; top - initial state. Only six independent stress components are shown.

\section{Discussion}

Fibre compression has generally been ignored owing to the extremely high suppleness of a fibre structure. However, while it is known that this type of loading can lead to buckling failure even for such supple structures, this behaviour has not been well-described anywhere. We emphasise that the collapsed state of a fibre is not related to the material yield but is governed by the material and geometric 
stiffness of the fibre column. Thus, the use of optical fibres has been restricted by their performance under compression. The main outcome of the present study has been to evaluate the stress-strain state relationship in an optical fibre during buckling loading. We performed compressional tests for samples with lengths ranging from 5 to $100 \mathrm{~mm}$, while the critical buckling force was evaluated only for the length of $35 \mathrm{~mm}$. For long samples, we recorded only the post-buckling states. Our study has demonstrated that the difference between the analytical approach and the experimental approach follows a linear relationship. In other words, with increasing sample length, this difference increases linearly, starting at $11 \%$ and ending at $309 \%$. Thus, the form of the relationship between Eulers critical force as a function of length is preserved, although Eulers equation can be seemingly used only for fibres of limited lengths. The following may be the reasons for the difference between the analytical and experimental results: 1) geometrical imperfections of the fibres, 2) tension/compression asymmetries, or 3) anisotropy of the material. The role of lateral-bending deformations has been studied by 19 for composite laminates and recently by [20] for organic fibres. They showed that the critical load increases quadratically with the number of initial imperfections in the samples. The results from our finite element analyses also showed that the dominant stress component in the post-buckling state is the axial stress in the initial state, although the shear stress is not negligible. Several authors have shown that the stress-strain response of selected textile fibres differs under tension and compression 21-23. We hypothesise that this may influence the behaviour of short samples loaded by compression more than long fibres. However, this may also explain the reason for the enormous difference between the experimental results and the computational data. The elastic modulus used in equation (1) was obtained from an auxiliary uniaxial test. We emphasise that none of these parameters were tested on optical fibres. We observed no decrease in the optical functionality of the fibres in spite of great differences among the post-buckling shapes. It is difficult to compare our results with those from the literature. Several studies have focused on stress analyses of optical fibres to investigate stress-induced birefringence [2426. In these studies, the optical fibres are assumed to be loaded transverse to their cross-sectional areas. Suhir 27, 28, has developed an analytical model for short, dual-coated optical fibres supported by a continuous elastic foundation and subjected to a compressive force applied to the free end. However, this model is valid only for short fibres with free ends. Moreover, no information is provided about the stress distribution in the optical fibre during buckling. A key parameter in textile engineering is the bending stiffness of woven structures. This parameter is measured either on a macro scale or a meso scale [29 32. We expect the compressional behaviour of single fibres to have the same degree of importance as other mechanical parameters with respect to their use in various structures. We also recognise that our study has some limitations. For example, the sensitivity of the force-displacement gauges of the traction machine were limiting factors for the long samples; therefore, we were not able to evaluate the critical force over the whole range of sample lengths. Also, the finite element material model that we used was developed from the isotropic, elastic behaviour of an optical fibre, but this material model would have failed in modelling the non-reversible, post-buckling deformation states of these optical fibres. 


\section{Conclusion}

We have evaluated the compressive stability of optical fibres using both experimental and computational approaches. Both the buckling and the post-buckling states of an optical fibre play important roles in determining the stress distribution in a fibre and consequently influence the fibre's optical/mechanical quality.

Acknowledgements This work was supported by the Ministry of Education, Youth and Sports of the Czech Republic and the European Union - European Structural and Investment Funds in the frames of Operational Programme Research, Development and Education - project Hybrid Materials for Hierarchical Structures (HyHi, Reg. No. CZ.02.1.01/0.0/0.0/16_019/0000843).

\section{Conflict of interest statement}

The authors declare no conflict of interest.

\section{References}

1. Kort Bremer, Frank Weigand, Yulong Zheng, Lourdes Alwis, Reinhard Helbig, and Bernhard Roth. Structural health monitoring using textile reinforcement structures with integrated optical fiber sensors. Sensors, 17(2):345, feb 2017. doi: $10.3390 / \mathrm{s} 17020345$.

2. Wei Zheng. Polymer optical fiber for smart textiles. In Handbook of Smart Textiles, pages 1-14. Springer Singapore, 2015.

3. Carlo Massaroni, Paola Saccomandi, and Emiliano Schena. Medical smart textiles based on fiber optic technology: An overview. Journal of Functional Biomaterials, 6(2):204-221, apr 2015. doi: 10.3390/jfb6020204.

4. J. E. Ritter, J. M. Sullivan, and Karl Jakus. Application of fracture-mechanics theory to fatigue failure of optical glass fibers. Journal of Applied Physics, 49 (9):4779-4782, sep 1978. doi: 10.1063/1.325508.

5. H. H. Yuce, P. L. Key, and P. J. Lemaire. Lifetime predictions for optical fibers. In Optical Fiber Communication. OSA, 1991. doi: 10.1364/ofc.1991.thg1.

6. R. Hiskes. Improved fatigue resistance of high-strength optical fibers. In Optical Fiber Communication. OSA, 1979. doi: 10.1364/ofc.1979.wf6.

7. G. Scott Glaesemann. Design methodology for the mechanical reliability of optical fiber. Optical Engineering, 30(6):709, 1991. doi: 10.1117/12.55870.

8. Naoki Mitsugi, Hirotoshi Nagata, Masaru Shiroishi, Nobuhide Miyamoto, and Ryosuke Kaizu. Optical fiber breaks due to buckling: Problems in device packaging. Optical Fiber Technology, 1(3):278-282, jun 1995. doi: 10.1006/ ofte.1995.1019.

9. M.H. Reeve. Investigation of optical fibre buckling in loose-tube packaging. Electronics Letters, 14(3):47, 1978. doi: 10.1049/el:19780034.

10. C.P Chen and T.H Chang. Fracture mechanics evaluation of optical fibers. Materials Chemistry and Physics, 77(1):110-116, jan 2003. doi: 10.1016/ s0254-0584(01)00578-8.

11. S. P. Timoshenko, J. N. Goodier, and H. Norman Abramson. Theory of elasticity (3rd ed.). Journal of Applied Mechanics, 37(3):888, 1970. doi: $10.1115 / 1.3408648$. 
12. R. E. Hobbs, M. S. Overington, J. W.S. Hearle, and S. J. Banfield. Buckling of fibres and yarns within ropes and other fibre assemblies. Journal of the Textile Institute, 91(3):335-358, jan 2000. doi: 10.1080/00405000008659512.

13. Pierre Alliez and Andreas Fabri. Cgal. In ACM SIGGRAPH 2016 Courses on - SIGGRAPH 16. ACM Press, 2016. doi: 10.1145/2897826.2927362.

14. E. Riks. An incremental approach to the solution of snapping and buckling problems. International Journal of Solids and Structures, 15(7):529-551, 1979. doi: 10.1016/0020-7683(79)90081-7.

15. Satish Balay, S. Abhyankar, M. Adams, P. Brune, K. Buschelman, L. Dalcin, W. Gropp, B. Smith, D. Karpeyev, D. Kaushik, L. Curfman McInnes, K. Rupp, H. Zhang, and S. Zampini. Petsc users manual revision 3.7. Technical report, apr 2016.

16. Robert D. Falgout and Ulrike Meier Yang. hypre: A library of high performance preconditioners. In Lecture Notes in Computer Science, pages 632-641. Springer Berlin Heidelberg, 2002.

17. Vicente Hernandez, Jose E. Roman, and Vicente Vidal. Slepc. ACM Transactions on Mathematical Software, 31(3):351-362, sep 2005. doi: 10.1145/ 1089014.1089019.

18. Hans Petter Langtangen. A fenics tutorial. In Automated Solution of Differential Equations by the Finite Element Method, pages 1-73. Springer Berlin Heidelberg, 2012.

19. John W. Gillespie and R.Byron Pipes. Compressive strength of composite laminates with interlaminar defects. Composite Structures, 2(1):49-69, jan 1984. doi: 10.1016/0263-8223(84)90042-4.

20. A. Andres Leal, Joseph M. Deitzel, and John W. Gillespie. Assessment of compressive properties of high performance organic fibers. Composites Science and Technology, 67(13):2786-2794, oct 2007. doi: 10.1016/j.compscitech.2007. 02.003 .

21. J. H. Greenwood and P. G. Rose. Compressive behaviour of kevlar 49 fibres and composites. Journal of Materials Science, 9(11):1809-1814, nov 1974. doi: $10.1007 /$ bf00541750.

22. John B. Miles. Compression of oriented nylon parallel to fiber axis. Textile Research Journal, 41(2):108-113, feb 1971. doi: 10.1177/004051757104100204.

23. John B. Miles. Compressional behavior of textile fibers. Textile Research Journal, 30(5):408-409, may 1960. doi: 10.1177/004051756003000514.

24. K. Okamoto, T. Hosaka, and T. Edahiro. Stress analysis of optical fibers by a finite element method. IEEE Journal of Quantum Electronics, 17(10): 2123-2129, oct 1981. doi: 10.1109/jqe.1981.1070652.

25. Zhaoming Zhu and Thomas G. Brown. Stress-induced birefringence in microstructured optical fibers. Optics Letters, 28(23):2306, dec 2003. doi: 10.1364/ol.28.002306.

26. E. Suhir. Calculated stresses in dual coated optical fibers. Polymer Engineering and Science, 30(2):108-117, jan 1990. doi: 10.1002/pen.760300207.

27. E. Suhir. Lateral compliance and elastic stability of a dual-coated optical fiber of finite length with application to nano-rods embedded into low-modulus elastic media. In 2009 59th Electronic Components and Technology Conference. IEEE, may 2009. doi: 10.1109/ectc.2009.5074019.

28. E. Suhir. Elastic stability of a compressed cantilever beam on an elastic foundation with application to a dual-coated fiber-optic connector. International 
Journal of Engineering Science, 83:85-94, oct 2014. doi: 10.1016/j.ijengsci. 2014.06.005.

29. Zhao-Qun Du, Tianxian Zhou, Ni Yan, Shen Hua, and Wei-Dong Yu. Measurement and characterization of bending stiffness for fabrics. Fibers and Polymers, 12(1):104-110, feb 2011. doi: 10.1007/s12221-011-0104-6.

30. Forsstrom. Influence of fibre/fibre joint strength and fibre flexibility on the strength of papers from unbleached kraft fibres. Nordic Pulp and Paper Research Journal, 20(02):186-191, jun 2005. doi: 10.3183/ npprj-2005-20-02-p186-191.

31. Rong Han, Yue qi Zhong, and Ge Wu. Calculating bending stiffness of woven fabrics based on image analysis. In Mechanics and Mechatronics (ICMM 2015). WORLD SCIENTIFIC, oct 2015. doi: 10.1142/9789814699143_0015.

32. B. Olofsson. Bending stiffness of looped fabrics. Textile Research Journal, 37 (7):618-619, jul 1967. doi: 10.1177/004051756703700711. 